\title{
Rational Design of Helical Nanotubes from Self-assembly of Coiled-coil Lock Washers
}

Chunfu $\mathrm{Xu}^{1}$, Elizabeth R. Wright ${ }^{2}$, Anil Mehta ${ }^{1}$, Louise C. Ser-pell ${ }^{3}$, Xiaobing Zuo ${ }^{4}$, Joseph S. Wall ${ }^{5}$, and Vincent P. Conticello ${ }^{1}$

1. Department of Chemistry, Emory University, Atlanta, GA 30322, USA

2. RPA Integrated Electron Microscopy Core, Emory University, Atlanta, GA 30322, USA

3. School of Life Sciences, University of Sussex, Falmer, E. Sussex, BN1 9QG, UK

4. X-ray Science Division, Argonne National Laboratory, Argonne, Illinois 60439, USA

5. Brookhaven National Laboratory, Upton, NY, 11973, USA

Structurally defined materials on the nanometer length-scale have been historically the most challenging to rationally construct and the most difficult to structurally analyze. Sequence-defined polypeptides represent attractive candidates as design elements for construction of these nano-scale materials, in that correlations can be drawn between sequence and higher order structure. However, the diversity of sequence space and the current limitations of theoretical approaches to reliably define the relationship between sequence and supramolecular structure present a significant challenge to the de novo design of novel materials architectures. Herein, we define a principle for the design of protein-based assemblies that we designate the re-coding hypothesis, in which we suggest that the sequences of natively folded protein structures can be re-designed to accommodate the formation of structurally defined supramolecular assemblies. In support of this hypothesis, we introduce modifications into the sequence of a structurally characterized seven-helix bundle that promote the self-assembly of the helical protomers into fibrillar nano-tubes that are capable of binding shape-appropriate small-molecules in the central channel.

The crystal structure of GCN4-pAA (PDB ID: 2HY6), a de novo designed peptide derived from the leucine zipper region of the $S$. cerevisiae transcription factor GCN4, displays a discrete seven-helix bundle structure that constitutes the largest, freely standing coiled-coil oligomer that has been structurally characterized thus far (Figure 1A). The heptameric assembly defines a continuous central channel with an internal diameter of approximately $7 \AA$. Computational analysis using the program CASTp indicated the presence of an internal void volume of $1,880 \AA^{3}$ associated with the central channel. The presence of several hexane-1,6-diol molecules in the central channel of the heptamer indicates that it is capable of binding appropriately shaped small-molecules within the cavity. The GCN4-pAA structure provides the opportunity to design tubular peptide fibrils of defined internal dimension through self-assembly. In addition, crystallographic analysis of the GCN4-pAA structure indicated a single residue shift in registry between adjacent helices, which resulted in an overall displacement of seven residues (i.e., one coiled-coil heptad repeat) upon closure of the bundle structure (Figure 1A). In contrast, most coiled-coil structures have no corresponding shift in helix registry and result in blunt-ended helical bundles. The structure of the seven-helix bundle of GCN4-pAA resembles a screw (or lock washer), in which the displaced edges at the seam provide an add itional interface for complementary interactions between the coiled-coil protomers.

We hypothesized that if the sequence of the peptide was appropriately modified to promote end-to-end association between the complementary surfaces perpend icular to the super-helix axis of the lock washer structure, then the helical bundle would self-associate into a high aspect-ratio fibril with a continuous 
channel throughout the assembly that would correspond in lateral dimensions to that of the seven-helix bundle observed in the crystal structure of the original peptide. To test our hypothesis, peptide 7HSAP1 was synthesized and structurally characterized.

Circular dichroism spectropolarimetry showed strong $\alpha$-helicity with MRE values that exceed those of the control peptide GCN4-pAA under identical conditions. The solution of 7HSAP1 displayed a flow linear dichroism spectrum with a strong positive signal at $207 \mathrm{~nm}$, which indicates the formation of an extended assembly in which the $\alpha$-helices align parallel to the flow direction of the Couette cell. The LD results are consistent with the hypothesis that 7HSAP1 assembles in solution to form extended arrays that stack along the direction of the super-helical axis (Figure 1B). STEM and Cryo-TEM of solutions of 7HSAP1 demonstrated the presence of fibrils of circa $3 \mathrm{~nm}$ in diameter. The observed diameter of the majority of 7HSAP1 fibrils compared well with the diameter of $3.1 \mathrm{~nm}$ that was determined from the crystal structure of GCN4-pAA. Solid-state NMR measurements on the 7HSAP1 fibrils were consistent with an assembly based on seven-helix bundles with displaced edges. In x-ray fiber diffraction, the meridianal reflections observed at 5.1 and $10.1 \AA$ are consistent with the regular repeat that arises from $\alpha$-helices and the position on the meridian supports the view that the $\alpha$-helices are aligned parallel to the fiber axes. Solid-state NMR measurements on the isotopically labeled 7HSAP1* fibrils were consistent with an assembly based on seven-helix bundles with displaced edges. SAXS/WAXS data support the presence of nanotube assemblies in solutions of 7HSAP1 with lateral dimensions that approximate those observed in the crystal structure of the seven-helix bundle of GCN4-pAA.

\section{References:}

[1] Liu, J.; Zheng, Q.; et al, P Natl Acad Sci USA 2006, 103 (42), 15457-15462.
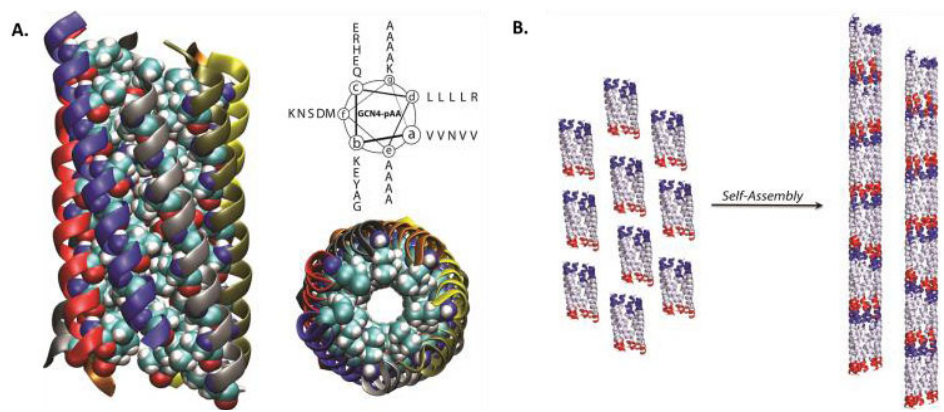

Figure 1. A. Crystal structure (PDB ID: 2HY6) of GCN4-pAA and helical wheel projection of the amino acid sequence of GCN4-pAA (upper right). B. Schematic representation of the proposed mode of self-assembly of lock washer structures derived from the 7-helix bundle of peptide 7HS AP1 into helical nanotubes.
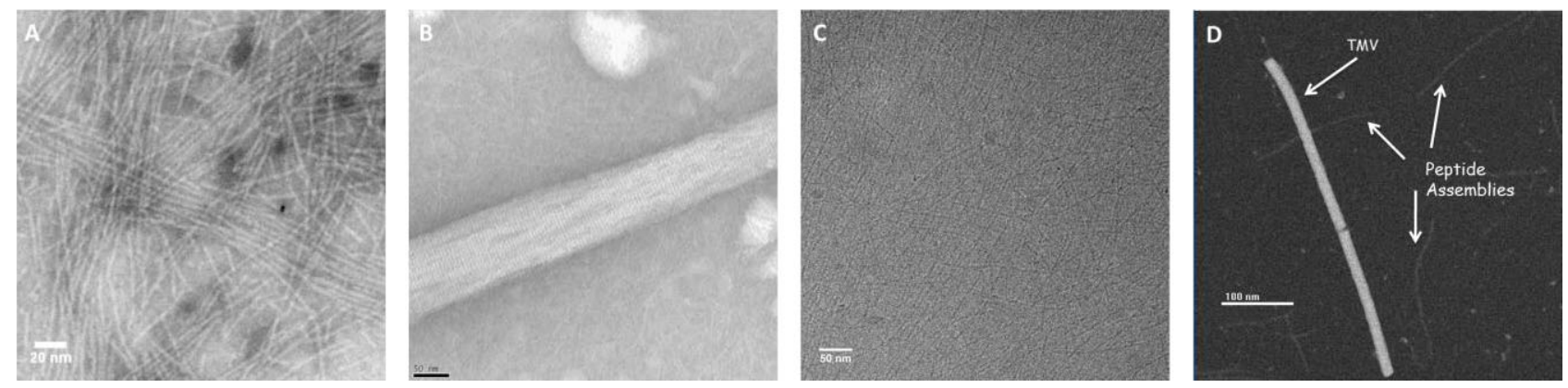

Figure 2. Electron microscopy of 7HS AP1 assemblies. A. Negative staining STEM. B. Conventional TEM. C. Cryo-EM. D. CryoSTEM. 